\title{
Stochastic Modeling of Central Apnea Events in Preterm Infants
}

Matthew T. Clark

John B. Delos

William \& Mary

Douglas E. Lake

Hoshik Lee

William \& Mary

et al.

\section{Recommended Citation}

Clark, Matthew T.; Delos, John B.; Lake, Douglas E.; Lee, Hoshik; and al., et, Stochastic Modeling of Central Apnea Events in Preterm Infants (2016). Physiological Measurement, 37(4), 463-484. https://doi.org/10.1088/0967-3334/37/4/463 accepted for inclusion in Arts \& Sciences Articles by an authorized administrator of W\&M ScholarWorks. For more information, please contact scholarworks@wm.edu. 
PAPER

\section{Stochastic modeling of central apnea events in preterm infants}

To cite this article: Matthew T Clark et al 2016 Physiol. Meas. 37463

View the article online for updates and enhancements.
Related content Quantification of periodic breathing in

Mary A Mohr, Karen D Fairchild, Manisha Patel et al.

- A new algorithm for detecting central apnea in neonates

Hoshik Lee, Craig G Rusin, Douglas E

Lake et al.

Neural networks andsleep monitoring

Azzam F G Taktak, Sarah Simpson, Sam Patel et al.

Recent citations

- Pre-Vent: the prematurity-related
$\frac{\text { ventilatory control study }}{\text { Phyllis A. Dennery et al }}$
- Advancing Continuous Predictive Analytics
Monitoring
Jessica Keim-Malpass et al




\title{
Stochastic modeling of central apnea events in preterm infants
}

\author{
Matthew T Clark ${ }^{1,7,9}$, John B Delos ${ }^{2}$, Douglas E Lake ${ }^{1,3}$, \\ Hoshik Lee $^{2,8}$, Karen D Fairchild ${ }^{4}$, John Kattwinkel ${ }^{4}$ \\ and J Randall Moorman ${ }^{1,5,6}$ \\ 1 Department of Medicine, University of Virginia, Charlottesville, VA 22904, USA \\ 2 Department of Physics, College of William and Mary, Williamsburg, VA 23185, \\ USA \\ 3 Department of Statistics, University of Virginia, Charlottesville, VA 22904, USA \\ ${ }^{4}$ Department of Pediatrics, University of Virginia, Charlottesville, VA 22904, USA \\ 5 Department of Biomedical Engineering, University of Virginia, Charlottesville, VA \\ 22904, USA \\ ${ }^{6}$ Department of Molecular Physiology, University of Virginia, Charlottesville, VA \\ 22904, USA
}

E-mail: clarkmt@vt.edu

Received 28 September 2015, revised 21 January 2016

Accepted for publication 22 January 2016

Published 10 March 2016

\begin{abstract}
A near-ubiquitous pathology in very low birth weight infants is neonatal apnea, breathing pauses with slowing of the heart and falling blood oxygen. Events of substantial duration occasionally occur after an infant is discharged from the neonatal intensive care unit (NICU). It is not known whether apneas result from a predictable process or from a stochastic process, but the observation that they occur in seemingly random clusters justifies the use of stochastic models. We use a hidden-Markov model to analyze the distribution of durations of apneas and the distribution of times between apneas. The model suggests the presence of four breathing states, ranging from very stable (with an average lifetime of $12 \mathrm{~h}$ ) to very unstable (with an average lifetime of $10 \mathrm{~s}$ ). Although the states themselves are not visible, the mathematical analysis gives estimates of the transition rates among these states. We have obtained these transition rates, and shown how they change with post-menstrual age; as expected, the residence time in the more stable breathing states increases with age. We also extrapolated the model to predict the frequency of very prolonged apnea during the first year of life. This paradigm-stochastic

\footnotetext{
${ }^{7}$ Current address: Advanced Medical Predictive Devices, Diagnostics, and Displays, Keswick, VA 22947, USA.

${ }^{8}$ Current address: Samsung Advanced Institute of Technology, Korea.

${ }^{9}$ Author to whom correspondence should be addressed.
} 
modeling of cardiorespiratory control in neonatal infants to estimate risk for severe clinical events - may be a first step toward personalized risk assessment for life threatening apnea events after NICU discharge.

Keywords: apnea, preterm infants, Markov model, ALTE

(Some figures may appear in colour only in the online journal)

\section{Glossary}

A Markov state representing apnea

$\alpha_{i} \quad$ Relative magnitude of exponential mode $i$

ABDX Apnea event lasting at least $X$ seconds with bradycardia and desaturation

ALTE Apparent life threatening event

$B_{i} \quad$ Markov state $i$ representing breathing

B Observation symbol probability density

C Transition probability density

K Transition rate matrix

$K_{\mathrm{ij}} \quad$ Transition rate from $i$ to $j$

$M \quad$ Number of distinct observables

$N \quad$ Number of Markov states

NICU Neonatal intensive care unit

$O_{k} \quad$ Observable $k$

$P \quad$ Transition probability

$P(t) \quad$ State probability vector at time $t$

PMA Post-menstrual age

$q(t) \quad$ Exponential probability distribution

$Q(t) \quad$ Exponential cumulative distribution

ROC Receiver operating characteristic

$S_{i} \quad$ Markov state $i$

$\mathrm{SpO}_{2} \quad$ Peripheral oxyhemoglobin saturation

$\tau_{i} \quad$ Expected value for exponential mode $i$

VLBW Very low birth weight, $<1500 \mathrm{~g}$

\section{Introduction}

Apnea of prematurity is the occasional breathing cessation and accompanying bradycardia and oxyhemoglobin desaturation that is experienced by nearly all infants born at very low birth weight $(<1500 \mathrm{~g}$, VLBW). While clinically significant apnea events usually resolve by 9 months from conception, apparent life-threatening events (ALTEs) occasionally occur after discharge from the neonatal intensive care unit (NICU) (Ramanathan et al 2001, Darnall 2009). Such prolonged apnea events have been postulated to be related to sudden infant death syndrome (SIDS), the leading cause of infant death after the neonatal period (Willinger et al 1991, Kinney and Thach 2009).

Foreknowledge of impending apnea in premature infants might lead to better management and earlier safe discharge. However, some clinical experience and scientific study suggest that prolonged apnea events may appear without warning (Ramanathan et al 2001, Darnall 2009), making their prediction and prevention impossible. On the other hand, it is known that apneas 
often appear in clusters, and these clusters of apnea events have been modeled as transient combinations of oscillatory breathing dynamics (Waggener et al 1982, 1984). The observation of clustering raises fundamental questions about the nature of neonatal apnea-is it a deterministic and predictable process, with a proximate cause for every individual apnea, or, on the other hand, does it result from a random or stochastic process? Control of breathing is maintained in the brain stem through gating of $\mathrm{CO}_{2}$ and $\mathrm{pH}$ sensitive ion channels - a mesoscale process at the borderline between true quantum unpredictability, (e.g. radioactive decay), and randomness in the sense of Laplace (1840), (that is, too many processes to account for) and we therefore reasoned that apneas might also have random kinetics. Indeed, stochastic switching of respiratory drive between normal rhythm and attenuated or abolished respiration has been demonstrated in animal models (Paydarfar and Buerkel 1995). Clustering of apneas viewed in this way takes on features of a random walk - the best predictor of whether an inebriated gentleman will appear beneath a solitary streetlight in the next few minutes is that he had appeared there a short time ago.

In this paper we develop a predictive statistical model based on the hypothesis that apneas can be described by a stochastic hidden-Markov model. The model works well, but nevertheless, we warn in advance that success of a stochastic model is not proof that apneas can only occur randomly, and such success should not deter search for deterministic models with better predictors of prolonged apnea. Indeed, algorithmic complexity theory indicates that distinction between random and deterministic mechanisms in long time series can rarely be achieved (Chaitin 1975).

In this paper, we study the kinetics of apnea events in the data set used to validate an apnea detection algorithm (Lee et al 2012). We begin by presenting the empirical distributions of apnea events and the time between events in a large, clinically-annotated database. We next build statistical models for neonatal breathing and apnea as a function of post-menstrual age (PMA) using a hidden Markov model-this type of model represents internal states of neonatal breathing as transitions among hidden states having varying degrees of stability. We present the concordant relation between model estimates and empirical values of apnea duration and burden. These parameters show steady development toward more stable neonatal breathing with PMA, represented by transitions in the models to favor more stable (longerlived) breathing states and away from the apnea and the proximal (shortest-lived) breathing state. Finally, we extrapolate the rates obtained from hidden Markov modeling to estimate the rate of substantial breathing cessations within the first year of life. We suggest that measuring transition rates for neonatal breathing control might identify infants at increased risk for prolonged, potentially-fatal apnea events.

\section{Materials and methods}

\section{Ethical approval}

The University of Virginia (UVA) Institutional Review Board approved this study under a waiver of consent. Computerized apnea detection results were not available to the patient care team, and decisions about apnea management and discharge readiness were based on nursing documentation of events and standard NICU monitor alarm data.

\section{Patient population}

We analyzed cardiorespiratory waveforms from 298 VLBW infants consecutively admitted to the UVA NICU between January 2009 and June 2011. Demographic information, including 
Table 1. Demographic characteristics of the population used to model apnea (2nd column) as a function of PMA and (3rd column) in the eight days prior to discharge home. Data are presented as median (25th, 75 th percentile).

\begin{tabular}{lll}
\hline Infants studied ... & As a function of PMA & $\begin{array}{l}\text { Prior to } \\
\text { discharge home }\end{array}$ \\
\hline$N$ & 298 & 196 \\
Gestational age (weeks) & $27(25,29)$ & $28(26,29)$ \\
Birth weight (grams) & $1010(788,1263)$ & $1025(795,1298)$ \\
Males & 143 & 108 \\
Discharged home & 223 & 196 \\
Died & 19 & 0 \\
Transferred & 56 & 0 \\
PMA at discharge (weeks) & $37(35,39)$ & $37(35,39)$ \\
Days in NICU & $52(26,93)$ & $62(36,93)$ \\
Days on mechanical ventilation & $3(0,29)$ & $2(0,24)$ \\
ABDs: apneas $>10$ s with bradycardia $(<100$ bpm) and & 26088 & 1577 \\
$\mathrm{O}_{2}$ desaturation $(<80 \%)$ & & 724 \\
ABDs $>10$ s with bradycardia $\left(<100\right.$ bpm) and $\mathrm{O}_{2}$ & N/A & \\
desaturation $(<80 \%)$, after additional filtering & & $0.7(0.1,2) \mathrm{a}$ \\
ABD events per patient per day of analyzable data & $1.48(0.37,3.4)$ & \\
\hline
\end{tabular}

${ }^{a}$ ABDs per day during last $8 \mathrm{~d}$ of stay.

birth weight, gestational age at birth, birth date, and types and times of respiratory support, were collected from patient medical records. Table 1 shows the demographic characteristics of the infants. Median gestational age at birth was 27 weeks and median birth weight $1010 \mathrm{~g}$. The number of infants discharged home includes those that were discharged with respiratory support. Figure 1 shows a histogram of the number of patients with data available at each PMA (gestational age plus age from birth), with distinction made by disposition at discharge.

We also analyzed patients within the $8 \mathrm{~d}$ preceding discharge to home: we found 196 VLBW infants that had data available for analysis immediately preceding discharge to home. Table 1 shows the demographic characteristics of this sample of infants.

\section{Data acquisition}

All patients in the UVA NICU have continuous cardiorespiratory monitoring. We collected signals from three electrocardiograph leads digitized at $240 \mathrm{~Hz}$, the chest impedance pneumograph digitized at $60 \mathrm{~Hz}$, and pulse oximetry digitized at $120 \mathrm{~Hz}$ from the GE bedside monitors using a central network server (Bedmaster Ex, Excel Medical, Jupiter, FL) behind the clinical firewall. We also collected heart rate, respiratory rate, and oxygen saturation vital signs derived by the monitor. Data were transmitted to our centralized computing and storage cluster and analyzed in parallel.

Of the total NICU stay for all patients (55 patient years) (Clark et al 2012), 51\% of data were deemed unsuitable for analysis due to epochs where recording was interrupted. Data were analyzed in 16 min epochs, and we required continuous time series of 3 ECG leads, chest impedance, and heart rate from the physiological monitor to define epochs as suitable for analysis. Missing chest impedance and dropped data packets from the data acquisition system were the most common causes for excluding an epoch. Of the remaining data (26.5 patient years), $10 \%$ were excluded to avoid the times during which the infants were being treated with mechanical ventilation. 


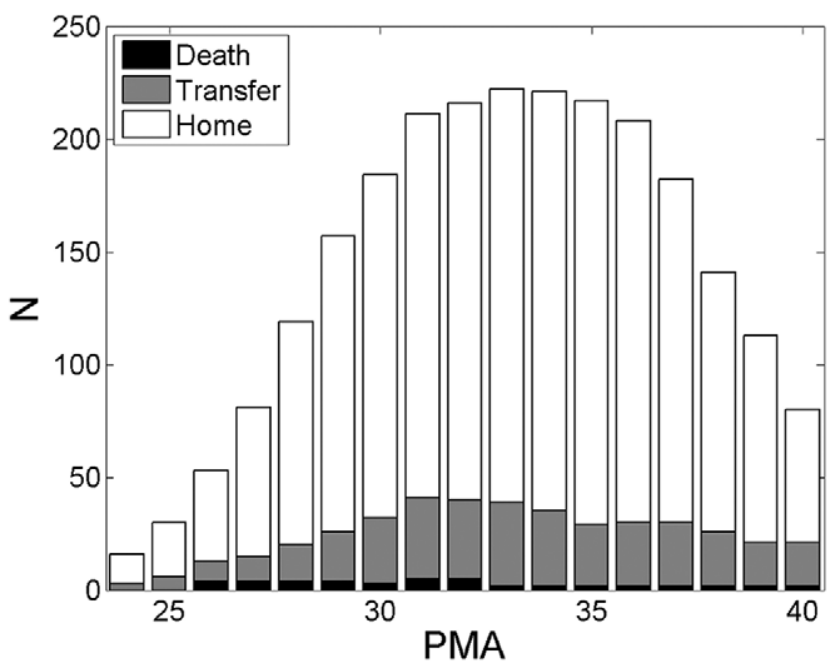

Figure 1. Patient data histogram. The histogram shows of the number of patients that had data available for analysis at each PMA. Distinction is made between infants who were discharged to home (white), those who were transferred to another unit or hospital (grey), and those who died (black). Only PMA up to term (40 weeks) are represented.

\section{Apnea detection}

Apnea events were identified in this data set as part of a previous study by our group-the apnea detection algorithm has been validated with accuracy $>90 \%$ by comparison with clinical inspection of waveform time series (Lee et al 2012, Vergales et al 2013). Briefly, apnea was detected as low variance epochs in the chest impedance pneumograph after notch filtering in heart-clock time to eliminate cardiac artifact and high-pass filtering to remove movement artifact. Heartbeats were detected using a threshold-based algorithm (Pan and Tompkins 1985) as implemented by Clifford and co-workers (Tarassenko et al 2001, Li et al 2008). For the current analysis, apnea was defined as breathing cessation of at least $10 \mathrm{~s}$ with both associated bradycardia and desaturations. We consider this definition for apnea clinically relevant because the breathing cessation caused both a decrease in heart rate and a decline in oxygen level in the blood - this is a stricter definition than that given by Finer et al (2006) in order to assure clinical relevance. The thresholds for bradycardia ( $\mathrm{HR}<100$, within 50 s from the beginning of the apnea or $25 \mathrm{~s}$ from the end of the apnea) and desaturation $\left(\mathrm{SpO}_{2}<80 \%\right.$, within $55 \mathrm{~s}$ from the beginning of the apnea or $38 \mathrm{~s}$ from the end of the apnea) are based on detailed inspection of 932 central apnea events by clinical personnel, see the appendix of Lee et al (2012). This apnea detection method is based on chest movement and therefore identifies only central apnea events (obstructive events are identified as breathing). While this algorithm does not explicitly exclude epochs of periodic breathing (Barrington and Finer 1990, Poets and Southall 1991), breathing pauses in periodic breathing epochs are shorter than $10 \mathrm{~s}$ and seldom induce sufficient bradycardia and desaturation to meet our thresholds (Khan et al 2005, Wilkinson et al 2007).

The results of Lee et al (2012) show an acceptable false positive rate (5\%) throughout a NICU stay. Near discharge, however, the rate of false positives may be higher due to the reduced apnea rate and increased noise due to movement. We filtered out false positive apnea events within $8 \mathrm{~d}$ of NICU discharge as detailed in the appendix, section Filtering out false positive apnea events near discharge. 
Table 2. Model parameters for time between apnea based on all patients at all times.

\begin{tabular}{lll}
\hline$B_{i}$ & Percent of distribution $(100 \alpha)$ & Mean time between apneas $\left(\tau_{i}\right)$ \\
\hline 1 & $12.9 \%$ & $31.9 \mathrm{~h}$ \\
2 & $59.5 \%$ & $3.9 \mathrm{~h}$ \\
3 & $16.4 \%$ & $16 \mathrm{~min}$ \\
4 & $11.1 \%$ & $9.7 \mathrm{~s}$ \\
\hline
\end{tabular}

\section{Empirical distribution of apnea and inter-apnea epochs}

We studied the duration and timing of 26088 apnea events occurring from admission through discharge for the patient population in table 1. Figure 2(a) shows the cumulative distribution of the apnea durations (dots). For each patient we identified sequential apnea events and calculated the time between each-the inter-apnea epochs. Inter-apnea epochs that started or ended at missing data were excluded as these are periods where apnea may occur but not be detected. Figure 2(b) shows the cumulative distribution of observed inter-apnea epochs for all infants regardless of PMA (dots).

\section{Statistical modeling of neonatal apnea}

We modeled apnea of prematurity as a Markov process based on the interpretation of apnea as the result of transitions through decreasingly stable breathing states. One type of Markov model is a Markov chain, which has a large or infinite number of states and transitions only between adjacent neighbors. These models have diffusion-like behavior and have been used to model the tail of inter-breath interval distributions in infants (Frey et al 1998). These models result in power law distributions that may be useful for fitting apnea durations (Frey et al 1998) but are not appropriate for fitting multimodal distributions (McManus et al 1988) - no single power law can fit the distribution of inter-apnea epochs in figure 2(b), thus no solvable diffusion or fractal model describes the statistics of apnea in VLBW infants.

In the second type of Markov model a small number of states are postulated, with transitions occurring randomly from each state to others. Generally transitions are allowed directly from every state to every other state. Models of this type are used in a wide range of fields, e.g. speech recognition (Lee and Jean 2013, Schafer and Jin 2014), disease tracking (Detilleux 2008, Robertson et al 2011), and ion channel recording (Colquhoun and Hawkes 1982). In ion channel models, states are defined for open, closed, and blocked channels and transition rates are determined empirically. Knowledge of these transition rates allows for determination of important behaviors, for example mean open lifetime, mean time between openings, and number of ion channel openings per cluster. This type of Markov modeling also has important applications in medicine (Sonnenberg and Beck 1993) and has been used to evaluate obstructive sleep apnea in adults (Kim et al 2009) and detect apnea of prematurity in short heart rate time series for preterm infants (Altuve et al 2011).

\section{Markov model parameter estimation}

Parameters of Markov models were derived following methods from analysis of single ion channel recordings (Colquhoun and Hawkes 1977, Colquhoun and Hawkes 1982). A Markov model is specified by the number of distinct observables $M$, the number of states $N$, the observation symbol probability distribution $\mathbf{B}=b_{j}$, the state transition probability distribution $\mathbf{C}$ or the related transition rate matrix $\mathbf{K}$, and the initial state distribution $\mathbf{P}(0)$ (Rabiner 1989). We 
(a)

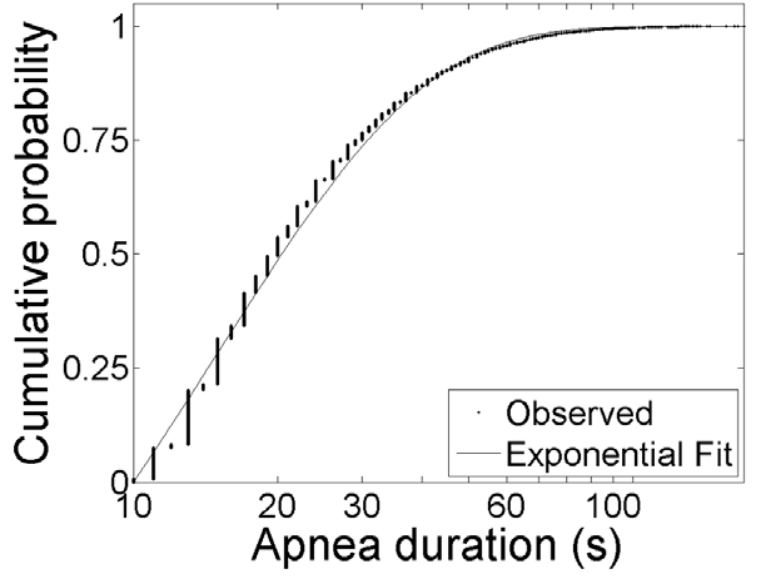

(b)

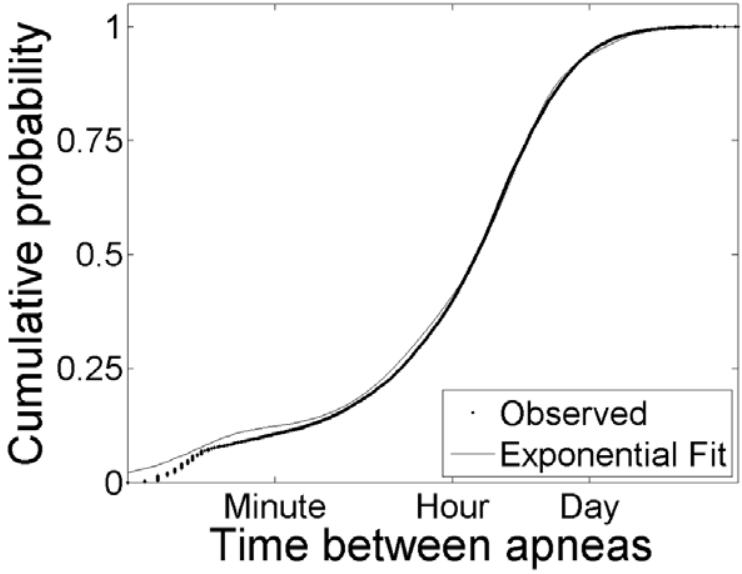

Figure 2. Statistics of apnea events. (a) Cumulative distribution of 26088 apnea durations. Vertical banding in observed data (dots) results from rounding of apnea durations to integer values. The distribution is well-described by an exponential distribution (lines). (b) Cumulative distribution of the logarithm of times between apnea. The data (dots) are well-described by a sum of four exponential distributions (line).

define $M=2$ distinct observables for neonatal breathing: breathing and apnea. Derivation of parameters $N$ and $b_{j}$ are given in the following section. Details on the remaining parameters are provided in the appendix.

\section{Neonatal breathing Markov model definition}

The number of states $N$ in a Markov model is chosen from study of the distribution of dwell times and transition rates (Sigworth and Sine 1987), in our case apnea durations and time between apnea events, the dots in figure 2. If a Markov model with discrete states and continuous time is correct, then the probability $q$ that an infant will stay in any given state for time $t$ with no transition to another state decreases with time exponentially, $q(t)=\exp (-t / \tau)$ where $\tau$ is the expected duration in the state. The corresponding cumulative distribution, which in 
our case is the probability that the duration is less than $t$, is given by $Q(t)=1-\exp (-t / \tau)$. Similarly, the probability that the infant will stay among $m$ hidden breathing states is a superposition of exponentials, and the corresponding cumulative distribution for the time between apneas is

$$
Q(t)=1-\sum_{i=1}^{m} \alpha_{i} \exp \left(-t / \tau_{i}\right)
$$

Here $\tau_{i}$ and $\alpha_{i}$ are the mean of the distribution and relative magnitude for exponential mode $i$, respectively.

The cumulative distribution of apnea durations was modeled with a single exponential, equation (1) with $\alpha=m=1$ (figure 2(a), solid curve), with parameter $\tau_{\mathrm{A}}$, the mean apnea duration, by maximizing the log likelihood of the parameter given the observed durations (Sigworth and Sine 1987). The mean apnea duration based on the model for apnea events greater than $10 \mathrm{~s}$ was $\tau=24.9 \mathrm{~s}$, and a single exponential function provided an accurate fit $\left(R^{2}=0.99\right)$.

In contrast, multiple distributions are evident in the cumulative distribution of inter-apnea epochs, figure 2(b) dots. We fit the data with increasing number of exponentials until the goodness-of-fit $R^{2} \geqslant 99.9 \%$. The goal was to minimize the complexity of the model (i.e. as few states as possible) while accurately fitting the data. We optimized the parameters using maximum likelihood and found that the sum of four exponentials, equation (1) with $m=4$ (figure 2(b), solid curve), adequately describes the data. The parameters are provided in table 2. Based on these results we defined the number of states in the Markov model for neonatal breathing to be $N=5$, including four breathing states and one apnea state, $S=$ $\left[B_{1}, B_{2}, B_{3}, B_{4}, A\right]$.

As stated above, the observables are that the infant is breathing or that the infant is not breathing, and the observation matrix $\mathbf{B}$ gives the probability of observing $O_{j}$ given that the model is in state $S_{i}$. For the apnea model this is trivial; the probability of observing breathing is 1 in each breathing state and 0 in the apnea state,

$$
B=\left[\begin{array}{ll}
1 & 0 \\
1 & 0 \\
1 & 0 \\
1 & 0 \\
0 & 1
\end{array}\right] .
$$

In order to characterize changes in breathing control with post-natal development, we created Markov models at weekly PMA — for each, we used a 3 week epoch centered on the one of interest. Specifically, we optimized the transition rate matrix $\mathbf{K}$ of a Markov model for each PMA from 25 to 40 weeks, 16 models in all. At each PMA a time series was created for each infant with data at that PMA, where each time series was a number each second: ' 0 ' if the infant is breathing and ' 1 ' if the infant is apneic. It is possible to have multiple time series per patient due to missing data or excluded epochs of mechanical ventilation. Each model was developed using $\mathbf{B}$ from equation (2) and the initial state distribution $\mathbf{P}(0)$ was taken to be,

$$
P_{i}(0)=\frac{\tau_{i}}{\sum_{i} \tau_{i}}
$$

where $\tau_{i}$ were determined using the exponential distribution fits to the data in figure 2 , and are listed in table 2. Note that the same observation matrix $\mathbf{B}$ and initial state distribution $\mathbf{P}(0)$ 
were used for each PMA and these were not allowed to vary during optimization. The transition rate matrix $\mathbf{K}$ for each PMA was determined using the optimization technique described in the appendix using the initial estimate from equation (A.4). The transition probability density $\mathbf{C}$ was calculated as the matrix exponential of the transition rate matrix $\mathbf{K}$.

\section{Transition rate confidence intervals}

At each PMA 40 bootstrap runs were completed (Feng et al 1996, Harrell 2001). For each run the $n$ patients at that PMA (see figure 1) were randomly resampled with replacement-for each run some patients were used multiple times and some patients not used. For each bootstrap run we calculated the transition rates using the same procedure described above and in the appendix. At each PMA, and for each transition rate, we calculated the $95 \%$ confidence interval by identifying the 2.5 and $97.5 \%$-tiles of the 40 samples-for 40 samples, the $95 \%$ confidence interval is defined by the minimum and maximum values.

\section{Results}

\section{Apnea in VLBW infants}

Inspection of the waveform data from bedside monitors revealed clusters of apnea separated by longer periods without apnea. Figure 3(a) shows a cluster of three clinically significant apneas within three minutes. Only the events labeled with black bars were identified as apnea by the algorithm (because, as stated above, the algorithm selects breathing cessations of at least $10 \mathrm{~s}$ accompanied by bradycardia and desaturation). Throughout, we use ABDX to indicate apnea events of at least $X$ seconds accompanied by bradycardia and oxygen desaturation. The first apnea is an ABD20. This is followed by two subclinical apneas, or breathing cessations that do not cause critical slowing of heart rate and declining oxygen saturation in the blood. Subsequently there are two clinical apneas; the first is $30 \mathrm{~s}$ and the second is $25 \mathrm{~s}$, an ABD30 and an ABD25. Figure 3(b) depicts the number of ABD10 events for a single preterm infant from 33 to 35 weeks PMA. Each half hour is represented by a vertical band, with the intensity of the band indicating the number of $\mathrm{ABD}$ events longer than $10 \mathrm{~s}$ during that time period (zero events per half hour is white, six is black). In this example apnea occurs at highly variable intervals; there are instances of multiple apneas within a half-hour as well as days without apnea. From day 21-24 there are no apnea events (the infant was not on mechanical ventilation during this time, and data were available more than $90 \%$ of the time). Subsequently, there is another cluster of apnea. These results suggested breathing states of varying stability, with apnea accessible from any state.

We also identified breathing cessations of substantial duration, many times longer than the $30 \mathrm{~s}$ duration characterized as ALTE (Ramanathan et al 2001). Figure 3(c) shows an example of an epoch of abnormal breathing control lasting nearly two minutes with bradycardia and oxygen desaturation. The detected event is labeled with a horizontal black bar. This epoch comes from an infant born at 27 weeks with a birth weight of $850 \mathrm{~g}$ who was eventually discharged to home from the NICU. This event was detected as an ABD110 by the automated detection algorithm, starting at $20 \mathrm{~s}$ and ending at $131 \mathrm{~s}$. It is important to note that, although this episode represents a period of apnea detected as an $\mathrm{ABD}$, isolated tiny fluctuations in the impedance signal may occur in long ABDs. This may permit these episodes to reach substantial lengths (Mohr et al 2014). 
(a)

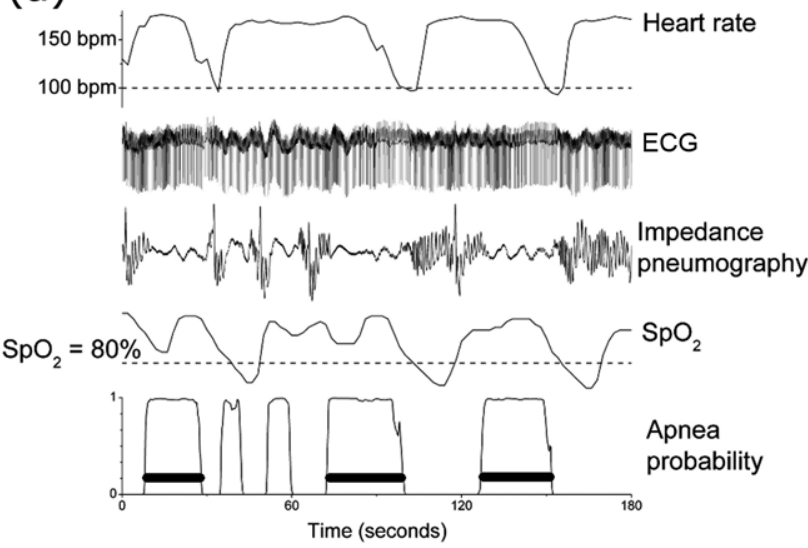

(b)

Apnea count

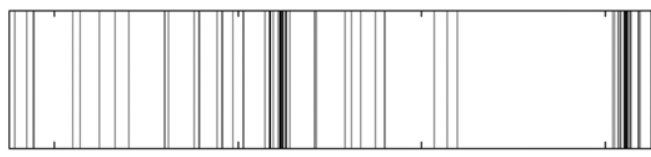

12

16

20

24

(c)

Days after admission
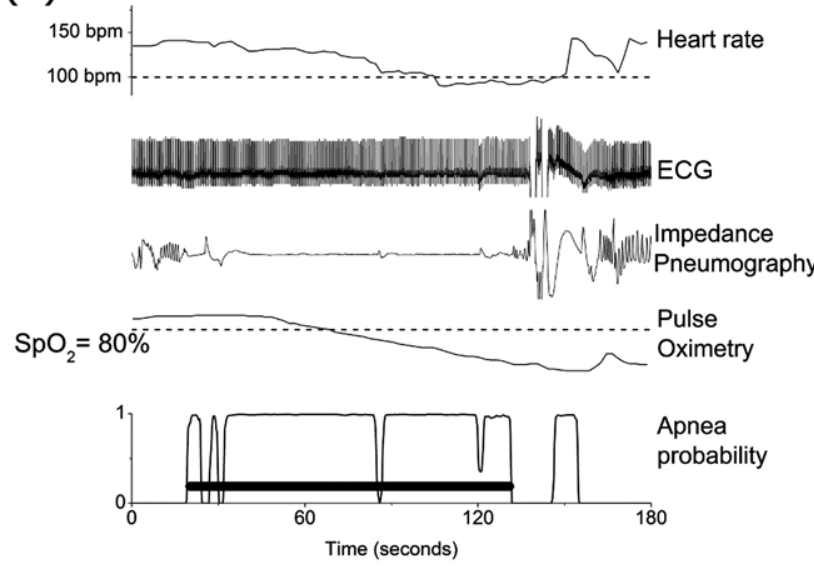

Figure 3. Physiological time series during apnea events. (a) Heart rate, ECG, impedance pneumography, peripheral oxygen saturation $\left(\mathrm{SpO}_{2}\right)$, and apnea probability for three minutes from a preterm infant. There is a cluster of three clinically significant apnea events within three minutes. (b) Time series of apnea events for a preterm infant over a two week period, from 33 to 35 weeks post-menstrual age. Each band represents the number of apnea events longer than $10 \mathrm{~s}$ in one half-hour; increasing density indicates more apnea. (c) Heart rate, ECG, impedance pneumography, peripheral oxygen saturation $\left(\mathrm{SpO}_{2}\right)$, and apnea probability for three minutes from a preterm infant. A prolonged period of apnea with associated bradycardia and oxygen desaturation lasting nearly two minutes is apparent. Isolated breaths during this episode may allow the prolongation. 


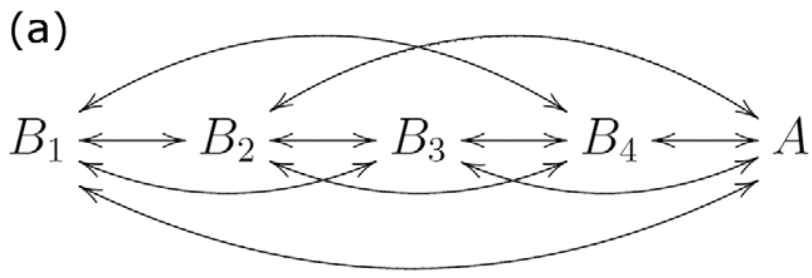

(b)

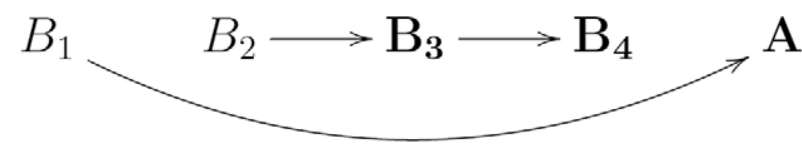

(c)

$$
B_{1} \longleftrightarrow \mathbf{B}_{2} \longleftarrow B_{3} \quad B_{4} \quad A
$$

Figure 4. Markov state diagrams. The diagrams show (a) all possible transitions, (b) 27 weeks PMA, (c) 40 weeks PMA. In (b) and (c) only the most probable transitions are shown. Major differences between residence times in the two cases are identified by bold symbols.

\section{Statistical models for central apnea in preterm infants}

We studied Markov models with the state diagram shown in figure 4(a), with one apneic state (A) and four breathing states $\left(B_{i}, i=1,2,3,4\right)$ numbered from most to least stable. For each PMA we optimized the transition rate matrix $\mathbf{K}$ of a Markov model. Figures 4(b) and (c) show the dominant transitions (arrows) and states (bold) for 27 and 40 weeks PMA, respectively. The important findings are that the earlier PMA has higher probabilities of transitions towards and residence in less stable breathing and apnea states. The model at higher PMA is characterized by larger probability of the second-most stable breathing state, and lower probability of apnea.

The Markov model transition rates are shown in figure 5 as a function of PMA. We found the average durations of these breathing states to be on the order of $10 \mathrm{~s}\left(B_{4}\right), 10 \mathrm{~min}\left(B_{3}\right), 2 \mathrm{~h}$ $\left(B_{2}\right)$, and $12 \mathrm{~h}\left(B_{1}\right)$, see figure 5 and appendix section Calculating apnea burden and mean apnea duration from a Markov model. Confidence intervals are shown for the transition rates and percent residence for each state.

The suitability of the model can be assessed by comparing its predictions to the observed data. We tested the goodness-of-fit of the model by comparing predicted and observed apnea burdens and the average duration of individual apneas. For each PMA, we calculated the apnea burden based on observed data as the number of seconds of apnea divided by the total monitoring time in days. We repeated the process for each Markov model being careful to censor apneas shorter than $10 \mathrm{~s}$ first, as apnea shorter than $10 \mathrm{~s}$ is not clinically significant (Finer $e t$ al 2006). Details are described in the appendix sections Calculating apnea burden and mean apnea duration from a Markov model and Censoring short apnea from the model. Figure 6 shows very good agreement between the predictions of the model and the observed data for apnea duration (figure 6(a)) and apnea burden (figure 6(b)). Observed data are shown as mean (circles) and 95\% confidence interval (error bars) for the sample of patients at each PMA. This agreement is evidence that a Markov model with five (four breathing and one apnea) states 
(a)

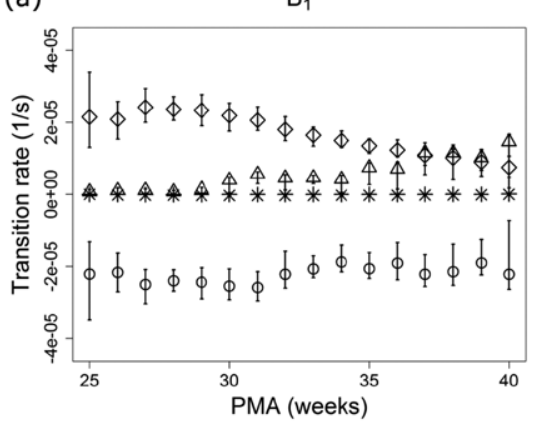

(c)

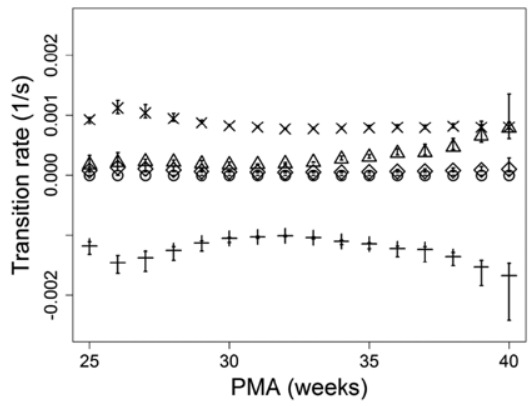

(e)

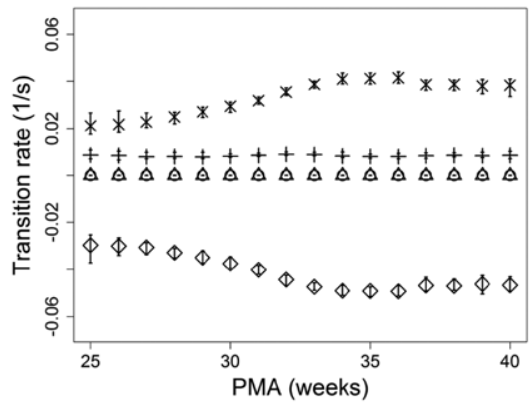

(b)

$\mathrm{B}_{2}$
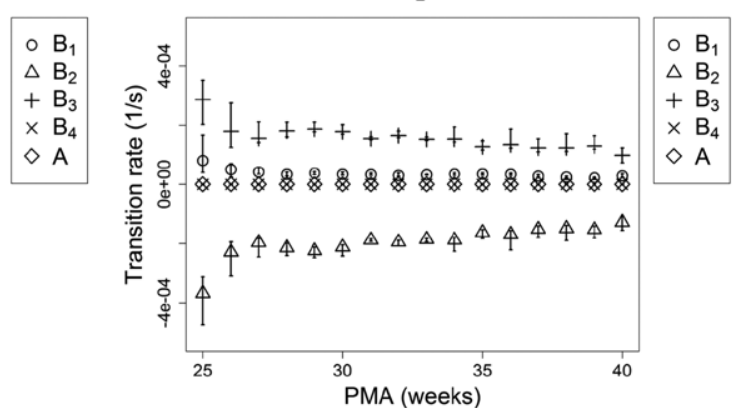

(d)

$\mathrm{B}_{4}$

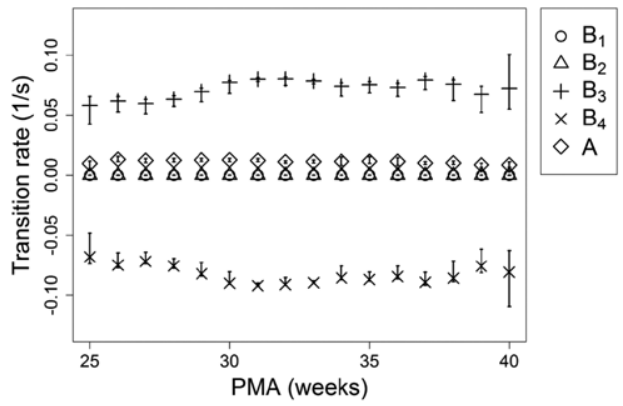

(f)

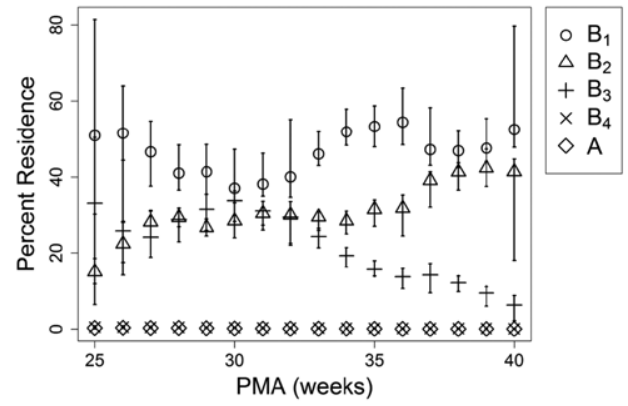

Figure 5. Transition rates versus PMA. Transitions are shown from $B_{1}$ (a), $B_{2}$ (b), $B_{3}$ (c), $B_{4}$ (d), and $A$ (e) into $B_{1}$ (circles), $B_{2}$ (triangles), $B_{3}$ (plusses), $B_{4}$ (crosses), and $A$ (diamonds) as a function of PMA. The percentage of time spent in each state is shown in (f) as a function of PMA. Segments above and below each symbol identify the $95 \%$ confidence interval and are sometimes smaller than the symbol.

and 2 observables (breathing and not breathing) can accurately characterize the kinetics of apnea in VLBW infants.

\section{The rate of severe apnea after NICU discharge}

Rare but clinically significant apnea events occur after discharge from the NICU. These ALTEs often lead to rehospitalization. We wish to estimate the rate of ALTE after NICU discharge. Thus we constructed a Markov model based on data from the eight days before discharge home, see table 1 . We optimized the transition rate matrix $\mathbf{K}$ of a Markov model using the 
(a)

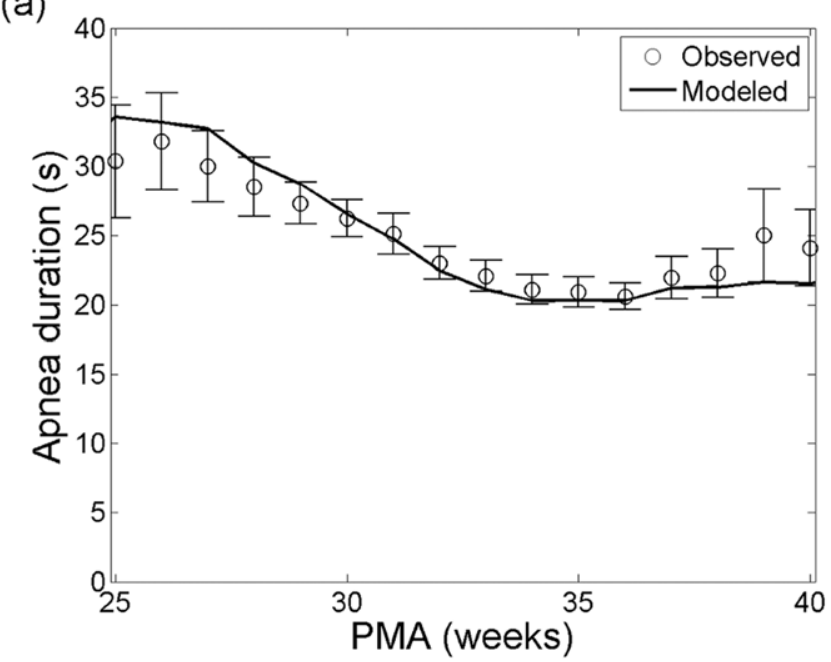

(b)

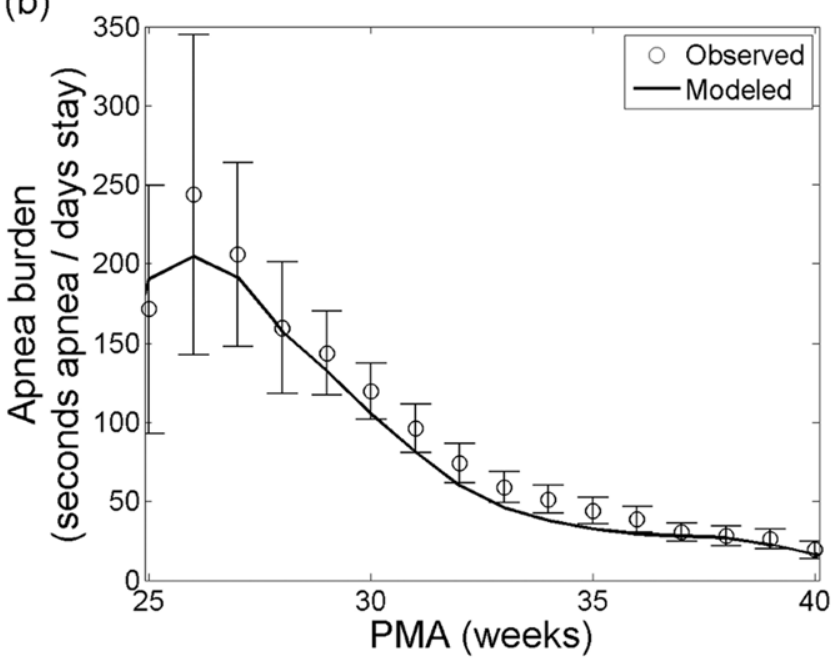

Figure 6. Apnea trends with PMA. Apnea duration (a) and apnea burden (b) as a function of PMA based on observation (circles) and modeling (solid). Error bars on the observations are $95 \%$ confidence intervals. The model results are adjusted to censor apnea events shorter than $10 \mathrm{~s}$.

same methods described above: the mean residence time in each Markov state in this model are $18.1 \mathrm{~h}\left(B_{1}\right), 2.2 \mathrm{~h}\left(B_{2}\right), 13.9 \mathrm{~min}\left(B_{3}\right)$, and $12.3 \mathrm{~s}\left(B_{4}\right)$.

We used the optimal transition rate constants from the discharge Markov model to estimate the rate of prolonged apnea after discharge. An analytical expression to estimate the rate of extreme apnea after NICU discharge is derived in the appendix section Estimating the rate of extreme apneas after discharge. This estimate is shown in figure 7-the figure addresses the question, 'given that an infant is discharged from the NICU to home, what is the probability that that infant will go $X$ days and have at least one apnea of duration at least $Y$ '. The rate of an apnea event is shown as a function of days after NICU discharge on the abscissa and duration on the ordinate. For example, our model estimates that the rate at which infants discharged to 


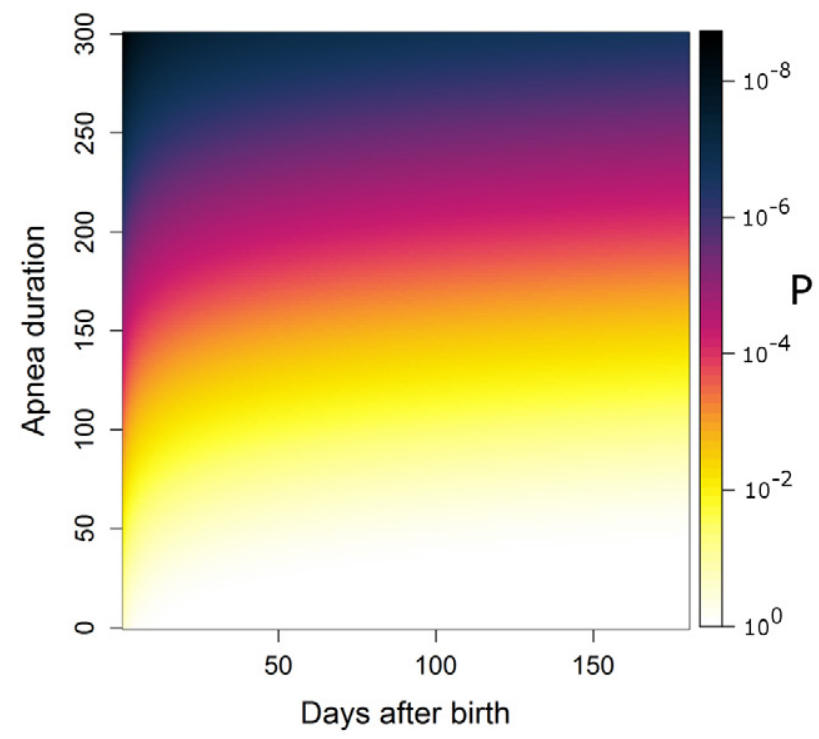

Figure 7. Apnea rate. The rate of apnea in post-neonatal patients based on a Markov model of all patients discharged home during their last week in the NICU. Grayscale is the probability of having at least one apnea with duration at least that on the ordinate between NICU discharge and the day on the abscissa.

home have at least one apnea $>128 \mathrm{~s}$ within the first 6 months after NICU discharge is 1 in 100. This extrapolation predicts that the rate of severe apnea $(\sim 180 \mathrm{~s})$ occurring after discharge is on the order of 1 per 1000. This is on the order of the occurrence rate of SIDS in the US population (Moon and the Task Force on Sudden Infant Death Syndrome 2011).

\section{Discussion}

We modeled neonatal apnea in VLBW infants in intensive care using stochastic Markov type models. The choice of a stochastic model was motivated by the simplicity of such models. This choice does not require that the underlying mechanisms of neonatal breathing control actually be stochastic as opposed to deterministic and high order. Our model accurately fit the trend of declining apnea duration and apnea burden with post-natal development in a large clinical dataset, and allowed for the observations of apnea bursts as well as long apnea-free epochs terminated by severe apnea events. We find that a small number of states, four breathing and one apnea state, are sufficient to accurately model apnea durations and the return time to apnea, and the development of these transitions with PMA. An interesting extension would be to develop models based only on inter-beat intervals, where bradycardia alone would act as surrogate for apnea with bradycardia and desaturation (Altuve et al 2011). Bradycardia duration and rate of descent are directly related to apnea duration and may be used to enhance such a model (Mohr et al 2014).

The longest breathing state, with transitions at about $12 \mathrm{~h}$, is the most frequently occupied state regardless of PMA and most preferably transfers to apnea until 38 weeks PMA, figure 5(a). In figure 5(f), it is interesting that the moderately stable state, $B_{3}$ with transitions at tens of minutes, is occupied $30 \%$ of the time until approximately 32 weeks PMA, after which it becomes less frequently occupied. Transitions from $B_{3}$ into more stable breathing state $B_{2}$ become increasingly likely with postnatal development in figure 5(c). These transitions may 
reflect the developing circadian and sleep cycles in neonatal brainstem physiology during this time. Circadian variations have been observed in the oxygen consumption of 31 week PMA infants (Bauer et al 2009), but this physiology may not measurably begin developing until after 32 weeks PMA (Chen 2010). The development of sleep-wake cycles has been observed as early as 28 weeks (Kuhle et al 2001, Olischar et al 2004), though sleep states develop in stability and occupancy over a longer period (Giganti et al 2006).

One speculative interpretation is that the most stable breathing state $B_{1}$ represents wakefulness, the second most stable state $B_{2}$ represents residence in various stages of sleep, and $B_{3}$ and $B_{4}$ represent transition states where the feedback from central and peripheral chemoreceptors to the respiratory centers in the neonatal brainstem may cause breathing to become unstable, leading to apnea. Early in neurological development the neonatal control centers do not vary oxygen consumption with day-night activity cycles and do not transition to stable sleep. This corresponds to the high probability of transition from the wakeful state $B_{1}$ into apnea, a frequently occupied unstable state $B_{3}$, and frequent transitions from unstable breathing $B_{3}$ into apnea before 29 weeks PMA (see figures 5(a), (c) and (f)). As circadian variation in oxygen consumption and control of breathing develop an infant spends more time in the stable sleep breathing state and less in unstable breathing, figure 5(f) starting at 30 weeks PMA. As sleep-wake cycles begin to develop transitions from wakefulness into stable sleep become more likely, figure 5(a) starting at 29 weeks PMA. Transitions out of stable sleep into unstable breathing ( $B_{2}$ to $B_{3}$, figure 5(b)) become increasingly likely to transition into another sleep cycle ( $B_{3}$ to $B_{2}$, figure $5(\mathrm{c})$ ). If these changing transition rates of Markov models are indicative of more stable sleep and circadian patterns approaching discharge, investigating the model for an individual patient may inform on that patient's postnatal brainstem development.

The rate of severe apnea events occurring after discharge from the NICU remains an unknown in neonatology. Though the data were collected during the $8 \mathrm{~d}$ prior to discharge from the NICU, we extrapolated our model to estimate the rate of such apparent life threatening events. Prolonged, potentially fatal apnea events after NICU discharge were studied by the Collaborative Home Infant Monitoring Evaluation (CHIME) study in the 1990s. A surprising result was the very high incidence of apneas exceeding $30 \mathrm{~s}$ accompanied by bradycardia and $\mathrm{O}_{2}$ desaturation that were not associated with obvious untoward effects (Ramanathan et al 2001). Our study also found clinically significant apnea events in the eight days prior to discharge home; many of these infants went home with respiratory support or on an apnea monitor. This suggests a great deal of resiliency in infants: our estimate of the rate of apnea lasting longer than $180 \mathrm{~s}$ during the first year of life is 1 in 1000, as shown in figure 7.

The implication is that information from breathing patterns might serve to identify infants that are more likely to experience prolonged potentially fatal apnea events during the first year of life. It is, counter-intuitively, the breathing durations and not the apnea durations that hold the information. Apnea durations were well-described by a single exponential decay function, a convincing argument for a single apnea state at all stages that allows for infrequent events of substantial duration. What is clinically important is how likely the current breathing state is to transition into apnea. On average, more stable breathing states are more likely with advancing development. A finding of improper maturation of transition rate constants might serve to identify the infants at highest risk for prolonged apnea after discharge to home.

\section{Limitations and strengths}

A limitation of our study is the inconsistent quality of neonatal breathing measurements-a large proportion of patient data were missing or not of suitable quality for analysis. More 
accurate and robust monitoring would improve the estimate for severe apnea occurring after NICU discharge, and would allow the stability of neonatal breathing control to be more accurately assessed. Another limitation is that additional filtering was performed only for apnea events within $8 \mathrm{~d}$ of discharge. Filtering all 26088 would likely increase the transition rates out of apnea in figure 5 and reduce the residual apnea burden and duration at higher PMA in figure 6. Another limitation is that, were data excluded due to movement, this may have led to a non-random distribution of missing data. If the more active infant is less likely to experience apnea, this would tend to skew our results towards shorter time periods between apneas. Whatever the imperfections of our algorithm, we emphasize that this continuous-monitoring system is far more accurate than nursing records.

Another limitation of our approach is that central apnea events are identified without distinction by cause. Some apneas near discharge may reflect poor sucking-swallowing-breathing coordination during feeds, or changing sleep architecture. Other normal physiologic processes contribute to apnea, notably anemia (Zagol et al 2012) and gastro-esophageal reflux (Poets 2004). Both of these normal physiologic phenomena may be progressing at the time of NICU discharge and may play a role in the stochastic distribution of apnea, with the possibility of very long time between apnea events as the chemoreceptors and other elements of the respiratory control system mature. Another limitation of our study is that we have considered only one of many manifestations of pathologic cardiorespiratory control. Future models for transitions between stable and abnormal cardiorespiratory control should include not only apnea (Martin et al 2002, Darnall 2009, Di Fiore et al 2013), but also, for example, periodic breathing (Khan et al 2005, Wilkinson et al 2007, Mohr et al 2015), cardiorespiratory uncoupling (Godin and Buchman 1996, Clark et al 2012), and transient heart rate decelerations (Dorostkar et al 2005, Moorman et al 2011). A final limitation is that we model the rate of prolonged apnea after discharge using data from the last $8 \mathrm{~d}$ in the NICU, thus extrapolating the data as if neurological development were complete at discharge.

A major strength of this study is the use of computer algorithm-detected apnea based on chest impedance waveforms rather than nursing documentation or a tally of bedside monitor alarms, thus allowing us to accurately quantify occurrence and duration of events (Southall et al 1983, Muttitt et al 1988, Vergales et al 2013). Automated detection algorithms could be continuously implemented at the bedside of preterm infants in intensive care, where all such infants already have routine cardiorespiratory monitoring. Such implementation would allow online estimation of the risk for apparently random transitions to isolated and severe apnea events following extended periods without apnea.

\section{Acknowledgments}

Funding: MTC, JBD, DEL, HL, JK, and JRM were funded by NIH GO grant 1RC2HD064488. JBD was partially supported by NSF grant 1068344.

\section{Competing interests}

JBD and HL have filed for a patent on the apnea detection algorithm.

\section{Data and materials availability}

Per the NIH requirements, data from this study are available through an MTA. 


\section{Author contributions}

MTC, DEL, and JRM designed the study and performed the analysis. JBD and HL developed the apnea detection algorithm and its refinement described in the appendix. KDF and JK performed validation of the apnea detection algorithm and its refinement, and provided clinical context for the results. All authors contributed to the writing of the manuscript.

\section{Appendix}

\section{Hidden Markov modeling}

Hidden Markov models represent time series data as random transitions between states that are not visible to the observer. At each instant the probability that an infant is in any one of $N$ states is described by a vector with $N$ components, $\mathbf{P}(t)=\left(P_{1}, P_{2}, \ldots, P_{N}\right)$. In the context of neonatal apnea, there are states that represent apnea and others that represent breathing states with varying levels of stability that are hidden from observers. The change of that probability vector is given by,

$$
\mathbf{P}(t+1)=\mathbf{P}(t) \mathbf{C}
$$

where $\mathbf{C}$ is a matrix that represents transition probabilities: the probability $\mathbf{P}$ that the system is in state $j$ at time $t+1$ given that it was in state $i$ at time $t$ is

$$
\mathbf{C}_{i j}=P(S(t+1)=j \mid S(t)=i)
$$

where $S(t)$ represents the state of the system at time $t$. In a stationary Markov model, this transition probability matrix does not depend on time. After each step, there is a finite probability that the infant remains in the same state $(i=j)$, and for each initial state $S_{i}$

$$
\sum_{j=1}^{N} C_{i j}=1 .
$$

The general theory of hidden Markov models includes also a collection of observables $\left(O_{k}, k=1,2, \ldots\right)$, and there is another matrix $\mathbf{B}$ that converts the state probability vector $\mathbf{P}(t)$ into the probability of observing each particular outcome $\left(O_{k}\right)$. In our case this matrix is trivial: the two outcomes are breathing or not-breathing. If an infant is in any of the breathing states the probability of observing breathing is 1 , while if the infant is in an apnea state the probability of observing breathing is 0 . Thus, 'hidden' refers to the fact that we cannot identify the stability of an infant's respiratory control system solely by observing that the infant is breathing.

\section{Maximum likelihood calculation of transition probabilities}

Several algorithms are available for obtaining maximum likelihood estimates of the onestep transition probability matrix $\mathbf{C}$ and from that its matrix logarithm $\mathbf{K}$. Here $\mathbf{K}$ is a timeinvariant $N \times N$ matrix of rate coefficients. The diagonal elements of $\mathbf{K}$ are negative, all others are positive, and each row of the matrix sums to zero. We used the Baum-Welch maximization algorithm to determine the optimal transition rate matrix K (Baum et al 1970, Juang and Rabiner 1991) as implemented in MatLab ${ }^{\circledR}$ (The MathWorks, Natick, MA), though the Viterbi algorithm is also an option (Shinghal and Toussaint 1979). These optimization algorithms allow for non-zero transition rates between any pair of states. This method requires an initial 
estimate of $\mathbf{C}$ and of the initial vector of probabilities $\mathbf{P}(0)$. The initial estimate $\mathbf{C}_{0}$ was derived from an initial estimate $\mathbf{K}_{0}$ that was chosen to be,

$$
K_{0}=\left[\begin{array}{ccccc}
-1 / \tau_{1} & 1 / \tau_{1} & 0 & 0 & 0 \\
1 / 2 \tau_{2} & -1 / \tau_{2} & 1 / 2 \tau_{2} & 0 & 0 \\
0 & 1 / 2 \tau_{3} & -1 / \tau_{3} & 1 / 2 \tau_{3} & 0 \\
0 & 0 & 1 / 2 \tau_{4} & -1 / \tau_{4} & 1 / 2 \tau_{4} \\
0 & 0 & 0 & 1 / \tau_{A} & -1 / \tau_{A}
\end{array}\right] .
$$

The transition rates were determined as a function of PMA using these parameters. The resulting coefficients of the transition rate matrix $\mathbf{K}$ are shown in figures 5(a)-(e). Note that the diagonal elements of $\mathbf{K}$ are negative to maintain a zero row sum. The coefficients are shown for the longest lifetime breathing state $B_{1}$ (a), the next longest lifetime breathing state $B_{2}(\mathrm{~b})$, the third longest lifetime breathing $B_{3}(\mathrm{c})$, the shortest lifetime breathing state $B_{4}(\mathrm{~d})$, and the apneic state $A$ (e). The percentage of time spent in each state is shown in figure 5(f).

\section{Calculating apnea burden and mean apnea duration from a Markov model}

To check the consistency between the model and the data, we estimate the apnea burden and the mean apnea duration. At steady state, the probability of apnea is the value of the corresponding element of the normalized left-eigenvector of the transition rate matrix $\mathbf{K}$ having eigenvalue equal to zero. If this probability is multiplied by the number of seconds in a day, it gives the apnea burden (seconds of apnea per days of monitoring) predicted by the Markov model. The average apnea duration predicted by the model is $-1 / K_{\mathrm{AA}}$, where $K_{\mathrm{AA}}$ is the transition rate from the apnea state into the apnea state. For any state $S_{i}$ the average residence time in that state is $-1 / K_{i i}$.

\section{Censoring short apnea from the model}

A Markov model assumes the dwell time in each state is exponentially distributed. Apnea durations are censored below $10 \mathrm{~s}$. Censoring an exponential probability distribution below a value $x$ has the effect of increasing the mean of that distribution by $x$. The average residence time in the apnea state $(A)$ must be decreased by $10 \mathrm{~s}$ to account for this fact. This is achieved by modifying transition rates $K_{\mathrm{A} i}, i=1 \ldots N$ representing transitions out of apnea as,

$$
K_{\mathrm{A} i}^{\prime}=\frac{K_{\mathrm{A} i}}{1-10\left|K_{\mathrm{A} i}\right|}
$$

\section{Filtering out false positive apnea events near discharge}

When modeling apnea during the $8 \mathrm{~d}$ prior to discharge home, the apnea detection algorithm produced a larger false positive rate than that during the rest of the stay. This is a result of the lower apnea rate and increased infant activity at this point in neonatal care. In order to reduce the rate of false positives and more accurately estimate the rate of apnea, we filtered events using an automated algorithm. For each apnea event we calculated two parameters: 


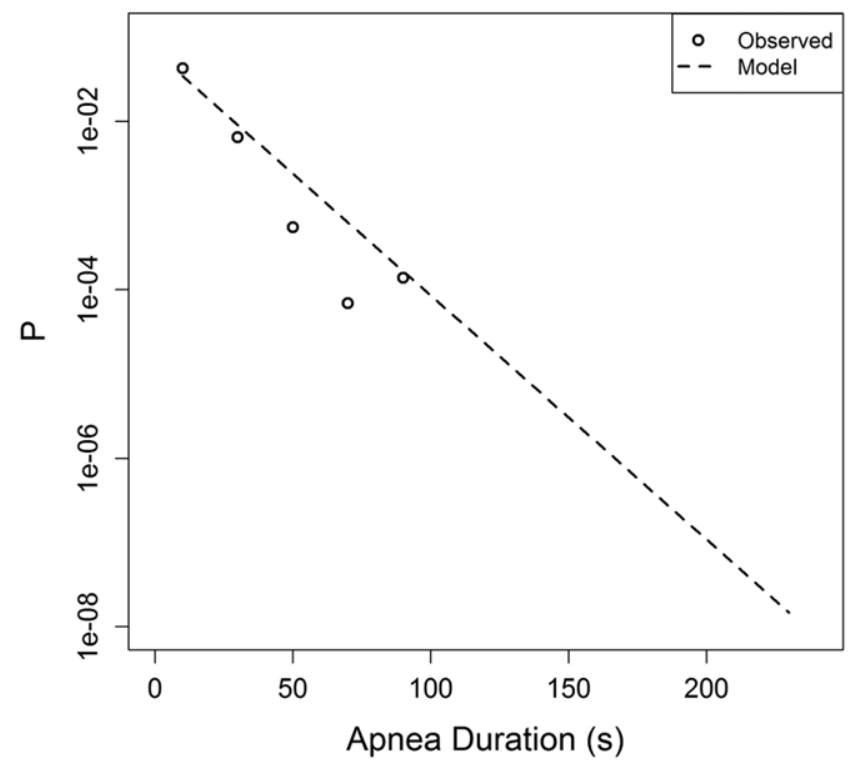

Figure A1. Probability density of automatically identified apnea durations during the eight days prior to discharge for 196 VLBW infants. Events longer than 60 s were identified as false positives by manual inspection, and were censored.

the area under the apnea probability (Lee et al 2012) divided by the apnea duration, and the power in the chest impedance between $0.5 \mathrm{~Hz}$ and $2.0 \mathrm{~Hz}$. These two parameters were calculated for 500 automatically-identified apnea events randomly selected throughout the entire stay of all infants in the study. Of these, 471 events were adjudicated as true apnea or false positive by agreement of two neonatologists. A logistic regression model was created using maximum likelihood estimation. The receiver operating characteristic (ROC) area of this model is 0.93 .

We used this model as an additional filter for apnea events, and determined a threshold below which apnea events were censored from our analysis. This threshold was determined by randomly selecting 100 events from the $8 \mathrm{~d}$ prior to discharge in 196 infants, of which 96 events were adjudicated. We selected a threshold for the apnea filter such that the false positive rate was equal to the false negative rate: we used an apnea filter threshold of 0.81 .

\section{Estimating the rate of extreme apneas after discharge}

We also computed a $\mathbf{K}$ matrix based on time series from the last $8 \mathrm{~d}$ prior to discharge home. We extrapolated the rate of apnea occurring after discharge from the NICU. We wish to estimate the probability that an infant will have at least one apnea event within $N$ days after discharge, and that at least one of those events will have duration greater than $d$. Figure A1 shows the distribution of apnea durations in the $8 \mathrm{~d}$ before discharge (circles) and the modeled distribution (dashed). The rate of apneas longer than about $1 \frac{1}{2} \mathrm{~min}$ is extrapolated.

We now model the probability of having $n$ apnea events in $N$ days using a Poisson distribution. We consider each day as a trial, and estimate the probability of $n$ 'successes' (apnea 
events) based on the expected number of successes $v$. In this context, the probability of having exactly $n$ apneas in $N$ days is the Poisson probability density distribution,

$$
P_{v}(n)=\frac{v^{n}}{n !} \mathrm{e}^{-v}
$$

Here $v$, the expected number of apneas in $N$ days, is

$$
v=-\alpha N K_{\mathrm{AA}} p_{\mathrm{A}} .
$$

where $\alpha=86400$ s per day, $K_{\mathrm{AA}}$ is the diagonal element of the rate matrix corresponding to the apneic state (i.e. the negative inverse of the mean apnea duration), and $p_{\mathrm{A}}$ is the probability of apnea. At steady state, this probability of apnea $p_{\mathrm{A}}$ is the value of the corresponding element of the normalized left-eigenvector of the transition rate matrix $\mathbf{K}$ having eigenvalue equal to zero. The probability that an apnea is shorter than $d$ seconds is the cumulative of the exponential distribution,

$$
P(D<d)=1-\mathrm{e}^{d K_{\mathrm{AA}}}
$$

The proposition that there are no apneas within $N$ days of discharge, or that any apnea events occurring within $N$ days are shorter than $d$, is,

$$
(n=0 \vee[n>0 \wedge \forall D: D<d])
$$

We use the distributions in equations (A.6) and (A.8) to find the probability of this proposition,

$$
P(n=0 \vee[n>0 \wedge \forall D: D<d])=\sum_{n=0}^{\infty} P(n) P(D<d)^{n} .
$$

This simplifies to,

$$
\begin{aligned}
& P(n=0 \vee[n>0 \wedge \forall D: D<d])=\mathrm{e}^{-\beta} . \\
& \beta=v \mathrm{e}^{d K_{\mathrm{AA}}} .
\end{aligned}
$$

The proposition of having at least one apnea with duration greater than $d$ is the negation of the proposition in equation (A.9),

$$
n>0 \wedge \exists D: D \geqslant d \equiv \neg(n=0 \vee[n>0 \wedge \forall D: D<d]) .
$$

The probability of prolonged apnea after discharge is therefore one minus the probability in equation (A.11),

$$
P(n>0 \wedge \exists D: D \geqslant d)=1-\mathrm{e}^{-\beta}
$$

This result is shown in figure 7 .

\section{References}

Altuve M, Carrault G, Beuchee A, Pladys P and Hernandez A I 2011 On-line apnea-bradycardia detection using hidden semi-Markov models Conf. Proc. IEEE Eng. Med. Biol. Soc. 2011 4374-7

Barrington K J and Finer N N 1990 Periodic breathing and apnea in preterm infants Pediatr. Res. $27118-21$

Bauer J, Janecke A, Gerss J, Masjosthusmann K, Werner C and Hoffmann G 2009 Circadian variation on oxygen consumption in preterm infants J. Perinat. Med. 37 413-7

Baum L E, Petrie T, Soules G and Weiss N 1970 A maximization technique occurring in statistical analysis of probabilistic functions of Markov chains Ann. Math. Stat. 41 164-71 
Chaitin G J 1975 Randomness and Mathematical Proof Scientific American 232 (5) 47-52

Chen A 2010 The circadian rhythm of expression of Bmal1 and Cry1 in peripheral blood mononuclear cells of preterm neonates J. Matern. Fetal. Neonatal Med. 23 1172-5

Clark M T et al 2012 Breath-by-breath analysis of cardiorespiratory interaction for quantifying developmental maturity in premature infants J. Appl. Physiol. $112859-67$

Colquhoun D and Hawkes A G 1977 Relaxation and fluctuations of membrane currents that flow through drug-operated channels Proc. R. Soc. B 199 231-62

Colquhoun D and Hawkes A G 1982 On the stochastic properties of bursts of single ion channel openings and of clusters of bursts Phil. Trans. R. Soc. B $3001-59$

Darnall R A 2009 Altes: still a puzzle after all these years J. Pediatr. 154 317-9

Detilleux J C 2008 The analysis of disease biomarker data using a mixed hidden Markov model Genet. Sel. Evol. 40 491-509

Di Fiore J M, Martin R J and Gauda E B 2013 Apnea of prematurity-perfect storm Respir. Physiol. Neurobiol. 189 213-22

Dorostkar P C, Arko M K, Baird T M, Rodriguez S and Martin R J 2005 Asystole and severe bradycardia in preterm infants Biol. Neonate $\mathbf{8 8} 299-305$

Feng Z, Mclerran D and Grizzle J 1996 A comparison of statistical methods for clustered data analysis with Gaussian error Stat. Med. 15 1793-806

Finer N N, Higgins R, Kattwinkel J and Martin R J 2006 Summary proceedings from the apnea-ofprematurity group Pediatrics 117 S47-51

Frey U, Silverman M, Barabási A L and Suki B 1998 Irregularities and power law distributions in the breathing pattern in preterm and term infants J. Appl. Physiol. 85 789-97

Giganti F, Ficca G, Cioni G and Salzarulo P 2006 Spontaneous awakenings in preterm and term infants assessed throughout 24-h video-recordings Early Hum. Dev. 82 435-40

Godin P J and Buchman T G 1996 Uncoupling of biological oscillators: a complementary hypothesis concerning the pathogenesis of multiple organ dysfunction syndrome Crit. Care Med. 24 1107-16

Harrell F E 2001 Regression Modeling Strategies: With Applications to Linear Models, Logistic Regression, and Survival Analysis (Berlin: Springer)

Juang B H and Rabiner L R 1991 Hidden Markov-models for speech recognition Technometrics 33 251-72

Khan A, Qurashi M, Kwiatkowski K, Cates D and Rigatto H 2005 Measurement of the $\mathrm{CO}_{2}$ apneic threshold in newborn infants: possible relevance for periodic breathing and apnea J. Appl. Physiol. 98 1171-6

Kim J W, Lee J S, Robinson P A and Jeong D U 2009 Markov analysis of sleep dynamics Phys. Rev. Lett. 102178104

Kinney H C and Thach B T 2009 The sudden infant death syndrome N. Engl. J. Med. 361 795-805

Kuhle S, Klebermass K, Olischar M, Hulek M, Prusa A R, Kohlhauser C, Birnbacher R and Weninger M 2001 Sleep-wake cycles in preterm infants below 30 weeks of gestational age. Preliminary results of a prospective amplitude-integrated EEG study Wien. Klin. Wochenschr. 113 219-23

Laplace P S 1840 Essai Philosophique sur les Probabilities (Paris: Paris Bachelier)

Lee L M and Jean F R 2013 Adaptation of hidden Markov models for recognizing speech of reduced frame rate. IEEE Trans. Cybern. 43 2114-21

Lee H et al 2012 A new algorithm for detecting central apnea in neonates Physiol. Meas. 33 1-17

Li Q, Mark R G and Clifford G D 2008 Robust heart rate estimation from multiple asynchronous noisy sources using signal quality indices and a Kalman filter Physiol. Meas. 29 15-32

Martin R J, Abu-Shaweesh J M and Baird T M 2002 Pathophysiology mechanisms underlying apnea of prematurity Neoreviews 3 e59-65

McManus O B, Weiss D S, Spivak C E, Blatz A L and Magleby K L 1988 Fractal models are inadequate for the kinetics of four different ion channels Biophys. J. 54 859-70

Mohr M A, Vergales B D, Lee H, Clark M T, Lake D E, Mennen A C, Kattwinkel J, Sinkin R A, Moorman J R, Fairchild K D and Delos J B 2015 Very long apnea events in preterm infants: systems failures in the Nicus J. Appl. Physiol. 118 558-68

Mohr M A, Fairchild K D, Patel M, Sinkin R A, Clark M T, Moorman J R, Lake D E, Kattwinkel J and Delos J B 2015 Quantification of periodic breathing in premature infants Physiol. Meas. 36 1415-27

Moon R Y and the Task Force on Sudden Infant Death Syndrome 2011 SIDS and other sleep-related infant deaths: expansion of recommendations for a safe infant sleeping environment Pediatrics 128 1030-9 
Moorman J R et al 2011 Mortality reduction by heart rate characteristic monitoring in very low birth weight neonates: a randomized trial J. Pediatr. 159 900-6 e1

Muttitt S C, Finer N N, Tierney A J and Rossmann J 1988 Neonatal apnea: diagnosis by nurse versus computer Pediatrics 82 713-20

Olischar M, Klebermass K, Kuhle S, Hulek M, Kohlhauser C, Rücklinger E, Pollak A and Weninger M 2004 Reference values for amplitude-integrated electroencephalographic activity in preterm infants younger than 30 weeks' gestational age Pediatrics 113 e61-6

Pan J and Tompkins W J 1985 A real-time QRS detection algorithm IEEE Trans. Biomed. Eng. 32 230-6

Paydarfar D and Buerkel D M 1995 Dysrhythmias of the respiratory oscillator Chaos 5 18-29

Poets C F 2004 Gastroesophageal reflux: a critical review of its role in preterm infants Pediatrics 113 e128-32

Poets C F and Southall D P 1991 Patterns of oxygenation during periodic breathing in preterm infants Early Hum. Dev. 26 1-12

Rabiner L R 1989 A tutorial on hidden Markov models and selected applications in speech recognition Proc. IEEE 257-86

Ramanathan R et al 2001 Cardiorespiratory events recorded on home monitors: comparison of healthy infants with those at increased risk for Sids JAMA 285 2199-207

Robertson C, Sawford K, Gunawardana W S, Nelson T A, Nathoo F and Stephen C 2011 A hidden Markov model for analysis of frontline veterinary data for emerging zoonotic disease surveillance PLoS One 6 e24833

Schafer P B and Jin D Z 2014 Noise-robust speech recognition through auditory feature detection and spike sequence decoding Neural Comput. 26 523-56

Shinghal R and Toussaint G T 1979 Experiments in text recognition with the modified viterbi algorithm IEEE Trans. Pattern Anal. Mach. Intell. 1 184-93

Sigworth F J and Sine S M 1987 Data transformations for improved display and fitting of single-channel dwell time histograms Biophys. J. 52 1047-54

Sonnenberg F A and Beck J R 1993 Markov models in medical decision making: a practical guide Med. Decis. Mak. 13 322-38

Southall D P, Levitt G A, Richards J M, Jones R A, Kong C, Farndon P A, Alexander J R and Wilson A J 1983 Undetected episodes of prolonged apnea and severe bradycardia in preterm infants Pediatrics 72 541-51

Tarassenko L, Clifford G and Townsend N 2001 Detection of ectopic beats in the electrocardiogram using an auto-associative neural network Neural Process. Lett. 14 15-25

Vergales B D et al 2013 Accurate automated apnea analysis in preterm infants Am. J. Perinatol. 31 $157-62$

Waggener T B, Frantz I D, Stark A R and Kronauer R E 1982 Oscillatory breathing patterns leading to apneic spells in infants J. Appl. Physiol. Respir. Environ. Exerc. Physiol. 52 1288-95

Waggener T B, Stark A R, Cohlan B A and Frantz I D 1984 Apnea duration is related to ventilatory oscillation characteristics in newborn infants J. Appl. Physiol. Respir. Environ. Exerc. Physiol. $57536-44$

Wilkinson M H, Skuza E M, Rennie G C, Sands S A, Yiallourou S R, Horne R S and Berger P J 2007 Postnatal development of periodic breathing cycle duration in term and preterm infants Pediatr. Res. 62 331-6

Willinger M, James L S and Catz C 1991 Defining the sudden infant death syndrome (Sids): deliberations of an expert panel convened by the National Institute of Child Health and Human Development Pediatr. Pathol. 11 677-84

Zagol K et al 2012 Anemia, apnea of prematurity, and blood transfusions J. Pediatr. 161 417-21 e1 